\title{
Tribological property study of xanthate acetic ester in hydrogenated oil
}

\author{
Juanhong LEI, Jian LIU, Liping XIONG, Xue YANG, Zhongyi HE* \\ School of Material Science and Engineering, Eastchina Jiaotong University, Nanchang City, China
}

*Corresponding Author: Zhongyi HE, Corresponding address, Nanchang City 330013, China; email address:hzy220567@163.com

\begin{abstract}
:
Xanthate was used as an initial reactant to synthesis two kinds of novel xanthate-containing acetic ester additives (HX and OX), and a four-ball machine was used as tribological tester to appraise their tribological behaviors in hydrogenated oil (10cst). The test results indicated that the as-synthesized additives possess good extreme pressure, antiwear and antifriction properties. The shorter carbon chains acetic ester, the better tribological properties the higher sulfur content. The action mechanism was estimated by analysis of the XPS (X-ray photoelectron spectroscopy) and SEM (scanning electron microscope) results of the worn steel ball surface, and the results illustrated that the protective film containing ferric sulfide and ferric sulfate compound was formed on the worn steel ball surface by xanthate acetic ester, and the protective film has good tribological properties.
\end{abstract}

Keywords: acetic ester derivative; additive; hydrogenated oil; tribological mechanism

\section{Introduction}

The modern industry needs better processing conditions, so it needs better lubricants in some processing fields. Many additives ${ }^{[1]}$ play a vital role in lubricating oil, because of its good guarantee in support of lubrication capability and satisfy the specific requirement. In many studies, it was reported that sulphur-containing ${ }^{[2]}$ compounds, such as sulfurized isobutylene, dibenzyldisulfide, etc, have the capability to improve lubricating oil extreme pressure performance. Sulfuration alkene is an excellent lubricant for offering effective protect to avoid mechanical fatigue, sintering and wear, so it is used in mechanical engineering oil, such as gear oil. Xanthate additive is a compound containing sulfur element, some research show that the binding energy of carbon-sulfur in xanthate is higher than that of sulfurized isobutylene, which makes the reaction activity of xanthate lower, therefore the xanthate is more stability in higher temperature condition and possesses lower corrosivity. The tribological properties of the target compound can be improved by adding xanthate groups to it. Some xanthate-containing triazine derivatives have been reported that they have outstanding tribological performance $^{[3]}$.

Two xanthate-containing ester target products were synthesized by using xanthate and chloroethyl chloroacetate as raw materials, the main purpose is to make the additive molecules have more xanthate functional groups. Their tribological behaviors of as-synthesis derivatives were appraised with a four-ball tribotester. The SEM and XPS analysis results were used to discuss their tribological mechanism in hydrogenated oil(10cst).

\section{Experimental details}

\subsection{Base stock and additive}

The base stock used is the hydrotreating oil produced in Daqing Refinery Factory, China. The hydrotreating oil (10cst) was without any pretreated before use, and it's $v_{40} \mathrm{C}$ is $51.71 \mathrm{~mm}^{2} \cdot \mathrm{s}^{-1}$, the flashpoint is $247^{\circ} \mathrm{C}$, viscosity coefficient is 126 .

The two xanthate acetic esters were synthesized according to the way in Scheme 1.

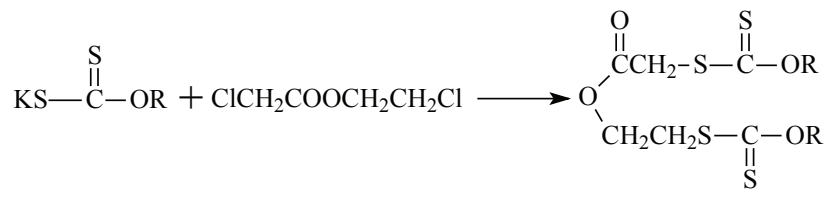

Where $\mathrm{R}=\mathrm{C}_{16} \mathrm{H}_{33}(\mathrm{HX}) ; \mathrm{C}_{8} \mathrm{H}_{17}(\mathrm{OX})$

Scheme 1. Reaction pathway of additives

The elemental analysis results of $\mathrm{HX}$ are as fellow: C is $63.88(63.33), \mathrm{H}$ is $10.09(10.00), \mathrm{S}$ is $17.19(17.78)$. And 
the elemental analysis results of $\mathrm{OX}$ are as fellow: $\mathrm{C}$ is 53.56(53.23), $\mathrm{H}$ is $8.11(8.06), \mathrm{S}$ is $24.99(25.81)$, the values in brackets are the theoretical analysis values. The test values of additives are basically the same as the theoretical values, the difference is within the experimental error range. Connecting with IR analysis results, it can be able to determine its structure.

\subsection{Testing apparatus and worn surface analysis}

Their tribological properties were evaluated with a four-ball tester, which according to the tribological testing standards (GB3142-82), similar to ASTM D-2783 ${ }^{[4]}$. The testing conditions are as below: rotating speed is $1450 \mathrm{rpm}$, the test lasted $30 \mathrm{~min}$ and room temperature. The used ball material is GCr15 bearing steel (AISI52100), and the hardness of the ball is 59-61 HRC. The average of wear scar diameters of three lower steel balls (code as WSD) was determined by an optical microscope, and its accuracy is $\pm 0.01 \mathrm{~mm}$. The friction coefficient is calculated according to the data recorded automatically of the four-ball tribotester.

After the test, ultrasonic cleaning of the upper steel ball was performed with petroleum ether ${ }^{[4]}$ for XPS analysis (PHI-5702 X-ray photoelectron spectrometer, USA), with the additive concentration of 1.0 wt.\% under $392 \mathrm{~N}$ for 30 min. Using the $\mathrm{MgKa}$ radiation as the excitation source, the binding energies of the target elements were determined at pass energy of $29.35 \mathrm{eV}$ and a resolution of $0.3 \mathrm{eV}$, using the binding energy of C1s $(284.6 \mathrm{eV})$ as the reference. The worn morphology analysis ${ }^{[5]}$ of the lower steel ball was performed by a JEM-1200EX type scanning electron microscope (SEM), to observe the wear mark on the rubbed surface.

\section{Results and discussion}

\subsection{The $P_{B}$ value}

The maximum non-seizure load $\left(\mathrm{P}_{B}\right.$ value $)$ of different content additives/10Cst is shown in Table1.

Table1 The $\mathrm{P}_{\mathrm{B}}$ value of oil samples in different additives contents

\begin{tabular}{crrrrr}
\hline \multicolumn{2}{c}{ Additives } & $0.0 \%$ & $0.5 \%$ & $1.0 \%$ & $1.5 \%$ \\
\hline \multirow{2}{*}{$\begin{array}{c}P_{B} \text { value } \\
(\mathrm{N})\end{array}$} & $\mathrm{HX}$ & 593.6 & 628.4 & 646.8 & 706.1 \\
\cline { 2 - 6 } \multicolumn{2}{c}{ OX } & 593.6 & 632.8 & 739.5 & 788.3 \\
\hline
\end{tabular}

From Table 1, the $\mathrm{P}_{B}$ values of $\mathrm{HX}$ and $\mathrm{OX}$ at any content are much higher than the $\mathrm{P}_{B}$ values of $10 \mathrm{cst}$, and the $P_{B}$ value increases when the additive concentration increase from $0.5 \mathrm{wt} \%$ to $1.5 \mathrm{wt} \%$. The $\mathrm{P}_{\mathrm{B}}$ values of $\mathrm{OX}$ at any content are higher than that of $\mathrm{HX}$, for the higher sulfur content than HX at the same condition. When the HX and OX concentration was $1.5 \%$, it can improve the $\mathrm{P}_{\mathrm{B}}$ values of 10 cst by $18.9 \%$ and $32.8 \%$ respectively, and this indicated that HX and OX have the excellent capacity in improving the load-carrying of base stock. The $\mathrm{P}_{\mathrm{B}}$ value is associated with sulfur content, the higher sulfur concentration, the better load-carrying performance of 10 cst.

\subsection{Friction-reducing Performance}

The friction coefficient curve of HX and OX in different contents under $392 \mathrm{~N}$, at $1.0 \mathrm{wt} \%$ content in different loads, were shown in Figure 1.
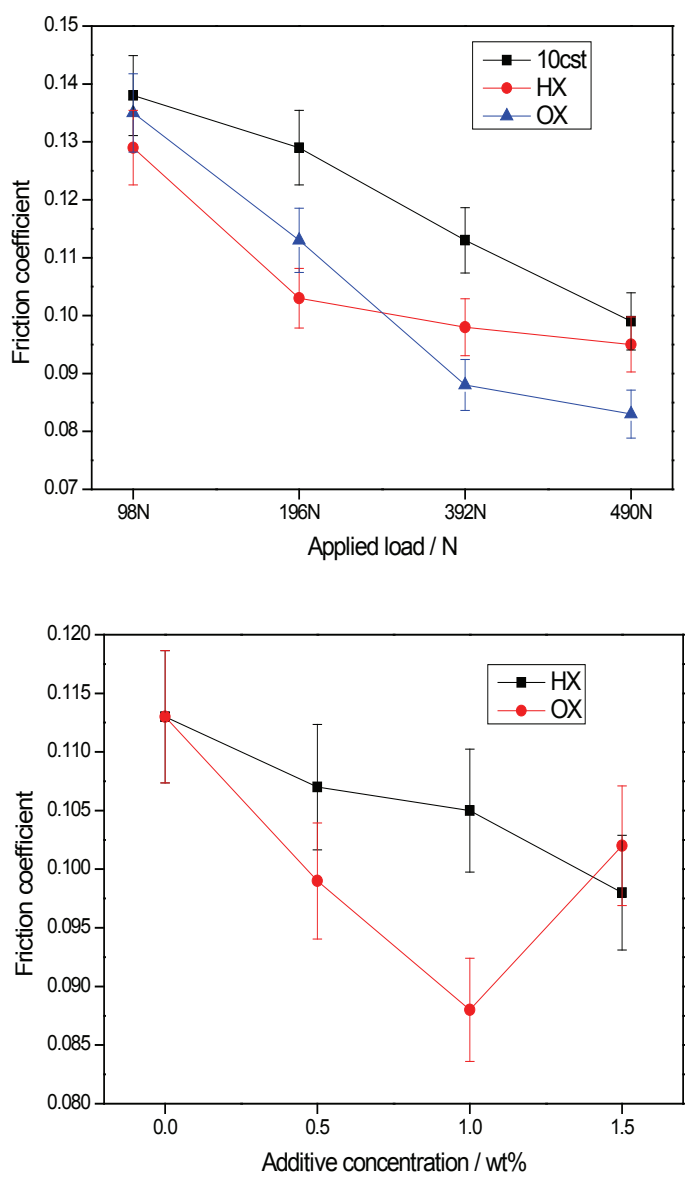

Figure 1 Friction coefficient of oil samples various applied load, additives concentration

From Figure 1(left), all the friction coefficient values of a lubricating stock decreased when the applied load increasing, meaning that the HX and OX possess good antifriction behavior under a wide load range. As shown in Figure 1(right), the applied load was $392 \mathrm{~N}$, the friction coefficient of $10 \mathrm{cst}$ was 0.114 . When $1.0 \mathrm{wt} \% \mathrm{HX}$ was added in $10 \mathrm{cst}$, the friction coefficient was 0.098 , declining by $13.3 \%$, and reduced $22.1 \%$ to 0.088 by adding $1.0 \mathrm{wt} \% \mathrm{HX}$ in $10 \mathrm{cst}$. And the antifriction performances of OX-containing oil samples are better than the HX-containing oil samples at the same additive content. With the higher amount of OX, the friction coefficient values of oil samples increased. The decrease of friction coefficient of oil samples can be attributed to the strength of the formed adsorption and/or tribochemistry reaction film caused by the HX (OX) on the lubricating interface ${ }^{[4]}$. When more $\mathrm{HX}(\mathrm{OX})$ was added to 10 cst, there were more additives molecules adsorbed on the metal surface, which can form thicker adsorption layer. More triboreaction products are generated in the metal surfaces to form a triboreaction film, and the triboreaction film also can prevent the asperities from direct contact, 
which made the friction coefficients smaller. When the content additives arrived to some degree, the physical and chemical adsorption and triboreaction process showed saturate tendency finally. It will cause corrosion worn when additive content over the better-adding concentration, then the friction coefficient will increase.

\subsection{Antiwear performance}

Figure 2 shows the relationship of WSD with additives concentrations at $392 \mathrm{~N}$, and applied load with $1.0 \%$ the concentration of additives in 10 cst.
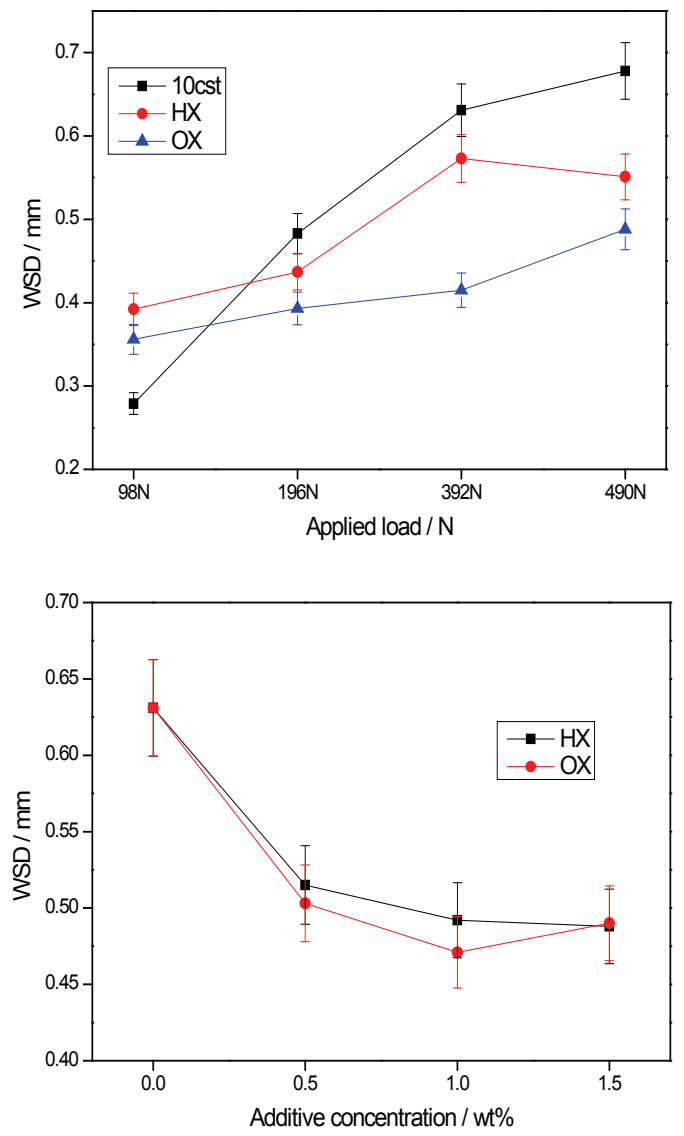

Figure 2 Variations in the WSD with concentration and applied load $(\mathrm{N})$

All HX and OX exhibit good antiwear properties in all applied loads. The WSD values increase as the applied load increasing from $98 \mathrm{~N}$ to $490 \mathrm{~N}$. The increase of WSD of base oil is the largest. The WSD value of OX and 10cst are both smaller than that of $\mathrm{HX}$ at the same content and applied load.

The xanthate-containing ester additive reduces the WSD of 10cst significantly, meanning that they have very good antiwear property. As the additive concentration increased, the WSD value decreased. The sulfur content increased when added more HX/OX additives in 10cst, and the antiwear capability was increased as well. It may be the result of the boundary protective film formed by HX/OX and/ or their decomposition products on the lubricating surface through tribochemistry reaction during the tribological process.

3.4 Discussion of the lubricating mechanism of $\mathrm{HX} / \mathrm{OX}$
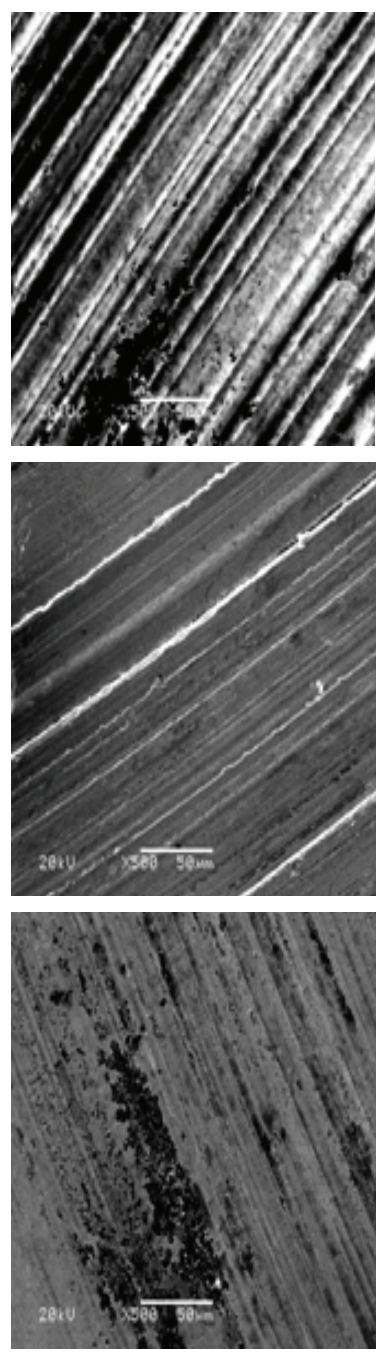

Figure 3 SEM of worn steel ball surface lubricated with 10Cst(left) and 1.0wt\% HX (middle); OX(right) at 392N, $30 \mathrm{~min}$

The SEM photographs are shown in Figure 3. When there are only lubricated with $10 \mathrm{cst}$, severe scuffing occurs on the worn steel ball surface, with the grain abrasion characteristic $^{[6]}$. There have only friction tracks to a small extent on the steel ball surface as lubricated with HX and $\mathrm{OX}$, and there existed some black cavities on the surface, which may be due to the corrosive worn made by sulfur element. It is because that there exist tribolochemical reactions between steel ball surface and sulfur element in $\mathrm{HX} / \mathrm{OX}$, to generate tribochemisorption ${ }^{[3]}$ during the lubricating process. The corrosive degree of steel ball lubricated by OX is bigger than that of HX, for the higher sulfur contents of OX. From the above analysis, it can be seen that the tribological performances of $\mathrm{HX}$ and $\mathrm{OX}$ are decided heavily by the ability of formed boundary protective film through adsorption (physisorption, chemisorption) and tribochemical reaction in the lubricating process.

In order to further explore the tribological mechanism 
of these kinds of xanthate acetic ester in 10cst, XPS analysis was used to analyze the worn steel ball surface lubricated by $1.0 \mathrm{wt} \% \mathrm{HX}$ in $10 \mathrm{cst}$, and the analysis results of characteristic elements are shown in Figure 4.
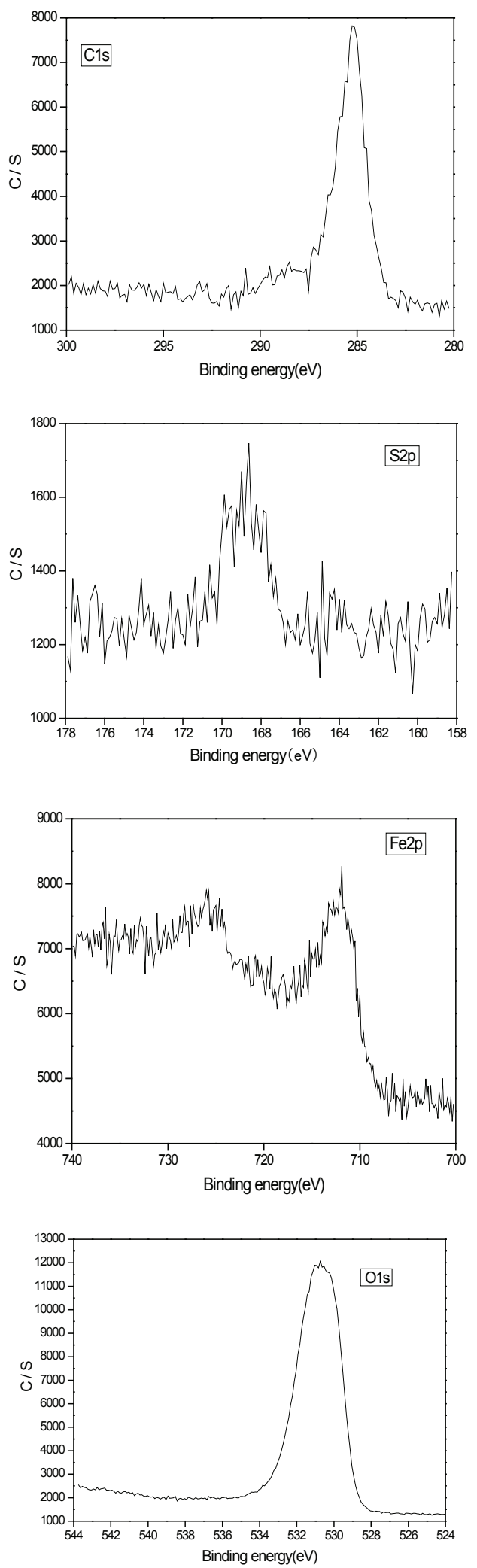

Figure 4 The XPS spectra of $\mathrm{C}_{1 \mathrm{~s}}, \mathrm{~S}_{2 \mathrm{p}}, \mathrm{Fe}_{2 \mathrm{p}}, \mathrm{O}_{1 \mathrm{~s}}(1.0 \mathrm{wt} \%$ HX /10cst)
The peak of $\mathrm{S}_{2 \mathrm{p}}$ spectrum ${ }^{[8]}$ was located at $168.8 \mathrm{eV}$ and $170.0 \mathrm{eV}$, which belong to ferric sulfide and ferric sulfate respectively. The formation of ferric sulfide and ferric sulfate indicates the tribochemical reaction had taken between HX with the metal surface in the lubricating process. The $\mathrm{Fe}_{2 \mathrm{p}}$ peak located at $711.3 \mathrm{eV}$ and $724.8 \mathrm{eV}$, which attributed to iron oxide and/or sulfide ${ }^{[9]}$, indicating that there existed oxidize or sulfurize on the lubricated steel surface because of the friction heat. The $\mathrm{O}_{1 \mathrm{~s}}$ peak located around $531.0 \mathrm{eV}$, belonging to iron oxide, and that means tribochemical reaction had occurred in the steel ball surface with HX during the lubricating process.

The SEM and XPS analysis results proved that the HX and OX may be decomposed to generate xanthate (or other sulphur-containing compounds $)^{[7]}$, so a relatively stable boundary lubrication film has been formed on the steel ball surface. The stable protect film is a complex lubricating film, which consists of adsorption layer and triboreaction layer. The triboreaction producing come from the tribochemical reaction between actively sulfur elements in the xanthate group and the freshly metal surface to form $\mathrm{FeSO}_{4}$, and / or $\mathrm{FeS}^{[10]}$, and the surface film possessing extreme pressure and antiwear capability. The surface film can avoid the direct contact of steel ball and other steel balls, so it can reduce the metal stock abrasion. Because of the stable reaction and adsorption film, the novel xanthate-containing ester can effectively increase the tribological performances of $10 \mathrm{cst}$.

\section{Conclusions}

From the above results, the following conclusions can be drawn:

(1) The synthesized ester derivatives as additives in 10cst base oil show excellent load-carrying performance at appropriate concentrations, and the $\mathrm{P}_{\mathrm{B}}$ values of $\mathrm{OX}$ at any content are higher than that of HX, for the higher sulfur content.

(2) The friction-reducing and antiwear capacities of $\mathrm{HX}$ and OX are highly susceptible to additive concentration and applied load. And the antiwear and friction-reducing behaviors of OX are better than that of HX at the same condition, for the different sulfur content.

(3) From the analysis results of SEM and XPS, it can be seen that the HX and OX have the behaviors of friction-reducing and antiwear in steel-steel sliding system through adsorption (physisorption, chemisorption) and tribochemical reaction with the steel ball surface. The formed boundary protective lubricating film during lubricating process helps to improve antiwear and frictionreducing capability of oil samples.

Author Contributions: Juanhong LEI and Zhongyi HE designed experiments; Jian Liu and Xue YANG carried out experiments; Liping XIONG analyzed experimental results. Zhongyi HE wrote the manuscript. All authors read and approved the manuscript.

Acknowledgements: The work was support by the National Natural Science Foundation of China (21563012, 
51962020); Jiangxi Science Foundation of China (20171BCD40009, 20181BAB203010); Jiangxi Provincial Education Department Foundation of China (GJJ180305).

\section{References}

[1] Wang Rulin, Lube Tribochemistry, China Petrochemistry Press, 1994, (in Chinese).

[2]. Igartua A, Fernández $X$, Areitioaurtena $O$, et al. Biolubricants and triboreactive materials for automotive applications[J]. Tribology International, 2009, 42(4):561568.

[3] Yinglei Wu, Zhongyi He, Xiangqiong Zeng, et al. Tribological and anticorrosion behaviour of novel xanthate-containing triazine derivatives in waterglycol[J]. Tribology International, 2017,110:113-124

[4] Dan Qiao, Haizhong Wang, Dapeng Feng. Benzimidazolyl phosphates as anti-wear additives in poly(ethylene glycol) for steel/steel contacts[J]. Lubrication Science, 2014, 26(1):1-11.

[5] Zhongyi He, Liping Xiong, Mingxue Shen, Sheng
Han, Jianqiang Hu, Wenyuan Xu, Tribological and antioxidation properties study of two N-containing borate ester derivatives as additive in rapeseed oil[J]. PLoS ONE,2018,13(12): e0207267

[6] Li Chaolong, Xiong Liping, Liu Hong, Xiong Liting, Wang Wei, Tribological Study of Xanthate- containing Acetic Ester as Additives in Hydrogenated Oil[J]. Applied Mechanics and Materials,236-237:123 - 127.

[7] Xiong Liping, He Zhongyi, Han Sheng, et al. Tribological properties study of $\mathrm{N}$-containing heterocyclic imidazoline derivatives as lubricant additives in waterglycol[J]. Tribology International, 2016, 104:98-108

[8] Yongjian Gao, Zhijun zhang, Qunji Xue, Study on 1,3,4-thiadiazole derivatives as novel multifunctional oil additives. Materials Research Bulletin. 1999;34:18671874.

[9] K.Komvopoulos, V.Chiaro, B.Pakter, et al, Antiwear tribofilm formation on steel surface lubricated with gear oil containing borate, phosphorus, and sulfur additives, Tribology Transactions. 2002;45(4):568-575. 